Article

\title{
Computational Study of the Effects of Processing Parameters on the Nonlinear Elastoplastic Behavior of Polymer Nanoclay Composites
}

\author{
Arifur Rahman and Xiang-Fa Wu * \\ Department of Mechanical Engineering, North Dakota State University, Fargo, ND 58108, USA; \\ Arifur.Rahman@ndsu.edu \\ * Correspondence: xiangfa.wu@ndsu.edu; Fax: +1-701-231-8913
}

Received: 29 September 2017; Accepted: 7 December 2017; Published: 9 December 2017

\begin{abstract}
Processing parameters (e.g., exfoliation extent and volume fraction) of clay particles in polymeric resins play a crucial role in the mechanical properties of polymer nanoclay composites (PNCs). This paper is aimed to investigate the effects of clay aspect ratio and volume fraction on the global mechanical properties (e.g., effective stiffness, yield strength, and ultimate tensile strength) of PNCs. During the process, computational micromechanics models are adopted to simulate the nonlinear elastoplastic behavior of the PNCs of varying clay particle volume fractions and aspect ratios subjected to uniaxial tension. A representative volume element (RVE) of the PNCs is employed for the finite-element-method (FEM) based computational simulations. The polymeric matrix is treated as an idealized elastoplastic solid with isotropic hardening behavior, and the clay particles are treated as stiff elastic platelets distributed evenly in the stack and stagger configurations in the matrix. Seven volume fractions $\left(V_{f}=0.5 \%, 1 \%, 2 \%, 5 \%, 7.5 \%, 10 \%\right.$, and $\left.15 \%\right)$ and seven aspect ratios (the ratio of platelet length over thickness $\rho=1,2,5,10,20,50$ and 100) of the reinforcing clay particles are utilized. Numerical experiments show that the effective modulus of the PNCs at small strains increases with the increase of either the clay volume fraction or the platelet aspect ratio largely following those predicted by classic micromechanics models. However, at the low particle aspect ratios (e.g., $\rho=1,2,5$ and 10), the ultimate tensile strength of the clay composite is nearly independent of the clay volume fraction up to $5 \%$ in the present study, i.e., the polymeric matrix governs the PNC strength; at the large particle aspect ratios (e.g., $\rho=20$ and 50), the ultimate tensile strength is significantly enhanced with growing clay volume fraction higher than $5 \%$ and reaches $\sim 150 \%$ of that of the polymeric matrix at $\rho=50$ and $V_{\mathrm{f}}=10 \%$. A comparative study is conducted for stack and stagger models for the prediction of the mechanical properties of PNCs. It shows that the stack model predicts slightly larger values of the effective stiffness and tensile strength than the stagger model. The numerical study shows that a large platelet aspect ratio through full exfoliation of the clay particles in matrix is crucial to achieving the preferable mechanical properties of PNCs as evidenced in experiments. The present results can be utilized to quantitatively explain the mechanical properties of clay particle-reinforced composites and PNCs within the framework of classic micromechanics, and provide guidelines for computer-aided nanocomposites design for processing property-tailorable PNCs.
\end{abstract}

Keywords: polymer nanoclay composites (PNCs); mechanical behavior; strength and stiffness; scaling analysis; elastoplastic; finite element analysis (FEA)

\section{Introduction}

Nanocomposites made of polymeric matrices reinforced with intercalated or exfoliated clay nanoparticles have become a focus of research in polymer composites in the last two decades after 
the seminal research initiated successfully by the Toyota research group in 1980s [1,2]. To date, substantial experimental investigations have shown that layered-silicate clay particles can be exfoliated into single nanosized platelets through ion exchange and can be well distributed in polymer melts or solutions as a nanoreinforcing phase in enhancing the mechanical and other physical properties of the resulting polymer nanoclay composites (PNCs). The most attractive evidence as demonstrated by numerous researchers worldwide is that layered silicate nanofillers can tremendously increase the tensile modulus, flexural stiffness and the tensile strength of PNCs at a filler weight content of only a few percent. Such preferable experimental observations are due mainly to the fact that the tensile modulus and strength of well aligned, exfoliated nanoclay platelets are close to the theoretical values of their perfect crystalline counterparts without defects (dislocations) and orders higher than those of the polymeric resins, as well as the high interfacial bonding strength (shear strength) between the clay nano platelets and the polymeric resins that exhaustively exploits the toughening effect of the clay nano platelets. For instance, nylon- 6 nanocomposites prepared through intercalative ring-opening polymerization of $\varepsilon$-caprolacetam modified montmorillonite to form fully exfoliated nano platelets can double the tensile modulus of the virgin nylon matrix at the filler weight content at $4.1 \%$ [3]; however, such giant modulus improvement cannot be achieved when the clay particles are dispersed in polymeric matrix to form intercalated polymer microcomposites [4]. Meanwhile, fully exfoliated clay nanofillers at a weight content of $\sim 5 \%$ can also enhance the tensile strength of the nanocomposites up to $50 \%$ as demonstrated in nylon nanocomposites that were prepared through in situ intercalative polymerization of $\varepsilon$-ecaprolactam in protonated aminododecanoic acid modified montmorillonite [4]. Besides, increasing experimental data have indicated that PNCs are capable of having desirable thermal stability, flame retardancy, and gas barrier properties that substantially broaden the applications of conventional polymer composites [4-10] in various industrial sectors. Among those, the most recent research investigated the effects of nanoclay morphologies (e.g., particle length, aspect ratio, bird nest structure, aggregation, etc. of halloysite and kaolinite nanoclay particles) on the thermal stability, surface wettability, and mechanical properties of biopolymer nanocomposite films for food packaging and other applications [11,12]. The studies showed that the thermal, tensile and surface wetting properties of the pectin resin can be noticeably improved by exfoliated halloysite nanotubes at relatively high weight concentration, which form unique bird nest structures in the pectin matrix of the resulting bionanocomposites. In addition, most recent research on the structural, physical and mechanical properties of nanoclay-reinforced fiber composites can be found in Reference [13].

Along with the enrichment of the database of experimental data of PNCs, remarkable efforts has been devoted to model-based understanding and numerical simulation of the mechanical behavior of PNCs for controllable processing and nanocomposites design. One typical treatment broadly considered by researchers is to consider PNCs as conventional particle-reinforced composites while an effective particle volume (size) is assumed [14]. Such treatment facilitates the utilization of classic micromechanics and numerical methods (e.g., finite element method-FEM) to analyze the mechanical behavior of PNCs [15]. With the assumption that the nanofillers are evenly distributed in the matrix, the effective mechanical properties, mainly the effective moduli, of the resulting nanocomposites can be approximately modeled by adopting Eshelby's equivalent model, self-consistent models of finite-length fillers, Mori-Tanaka type models, bound models, Halpin-Tsai model and its extensions, and modified shear-lag models, among others [15]. By comparison with these classic micromechanics models plus detailed finite element analysis (FEA), Tuchker and Liang concluded that the simple shear-lag model is capable of giving a good estimate of the longitudinal modulus $\left(E_{11}\right)$ of short-fiber reinforced composites when the fiber aspect ratio is greater than 10 [15]. Meanwhile, Tsai and Sun [16] developed a modified shear-lag model to investigate the load transfer efficiency in PNCs reinforced with intercalated and fully exfoliated clay particles, and their model was validated by using FEM. In addition, Weon and Sue [17] developed an experimental scheme to tailor the clay orientation and aspect ratio in nylon-6 PNCs through controlled shearing in order to study their effects on the mechanical properties. It was found that the effective modulus and tensile strength of the PNCs decreased with decreasing aspect 
ratio and alignment extent of the reinforcing clay nano platelets; in contrast, the fracture toughness and ductility of the PNCs increase simultaneously. In the study, the Halpin-Tsai and Mori-Tanaka micromechanics models were employed successfully to examine the dependency of composite moduli upon the orientation and aspect ratio of the clay platelets; the effective filler structure was established through mapping the effective structural parameters of fillers to the structural parameters of conventional fillers within the framework of micromechanics [18]. Similar studies were also conducted by many other researchers. For example, Zhu and Narh [19] performed detailed FEA of the mechanical behavior of aligned clay platelet-reinforced nanocomposites. In their model, the clay particles and the polymeric matrix were all treated as linearly elastic solids and an interlayer was proposed to model the transition region between the polymeric matrix and the fillers. Numerical simulations based on such a three-phase model showed that either decreasing interlayer modulus or increasing interlayer thickness resulted in decreasing effective moduli of the PNCs. In addition, Dai et al. [20] recently introduced an improved stagger model to analyze the stiffness and strength of PNCs reinforced with aligned, exfoliated clay nanoparticles; numerical results are close to those obtained in experimental studies. More recently, Dong and Bhattacharyya [21] performed finite element modeling to determine the effective moduli of polypropylene/organoclay nanocomposites. In the model, representative volume elements (RVEs) of stacking, staggering, and randomly distributed platelets in matrices were considered; their numerical results showed that the interlayer properties had less impact on the effective moduli of the PNCs reinforced with fully exfoliated clay nanoparticles. More detailed reviews on research progress in processing, characterization and modeling of the mechanical properties of PNCs can be found in the recent review papers in this topic, e.g., [4-6,13,21,22] and references therein.

In the above, it can be observed that the majority of the modeling studies reported in the literature was focused on the effective moduli of PNCs by adopting the classic micromechanics models and related computational methods within the range of linearly elastic deformation of the composites. Yet, no systematic study has been conducted on the ultimate tensile strength of PNCs though it is an important material parameter in practical applications of PNCs. In view of processing PNCs, the key processing parameters include the clay particle volume (or weight) fraction, exfoliation extent and platelet orientation in polymeric matrices, which dominate the mechanical properties of the resulting PNCs. Thus, in this study, we initiated a computational scaling study on the effects of processing parameters (i.e., the volume fraction and aspect ratio of the clay particles) on the effective modulus, yield strength, and ultimate tensile strength of PNCs by using a nonlinear FEM model. In the model, the typical stack and stagger RVEs of PNCs were adopted, and the polymeric matrix was treated as idealized elastoplastic solid with isotropic hardening behavior. A family of effective stress-strain diagrams was gained at varying volume fraction and aspect ratio of the clay particles through detailed nonlinear FEA. Dependencies of the effective stiffness, yield strength and ultimate tensile strength upon the processing parameters of the PNCs and relevant mechanisms were explored and discussed. Conclusions of the present study and relevant applications were addressed in the last section of the paper.

\section{Problem Statement and Simulation}

\subsection{Scaling Analysis of the Mechanical Properties of PNCs}

Given a PNC system, the processing parameters such as the particle volume (weight) fraction $V_{\mathrm{f}}$, aspect ratio $\rho$, defined as the ratio of the platelet length to the thickness in the simplified two dimensional (2D) case, and the platelet orientation angle $\theta$, among others, dominate the mechanical properties (e.g., effective modulus $E_{\mathrm{e}}$, yield strength $\sigma_{\text {ye, }}$, and ultimate tensile strength $\sigma_{\mathrm{ue}}$ ) of the resulting nanocomposite material. In this study, without loss of the generality, we adopted the 2D stack and stagger RVEs, in which all the clay platelets are assumed to be distributed either in stacked or staggered patterns in the polymeric matrix. According to the Buckingham $\pi$-theorem [23], the effective modulus $E_{\mathrm{e}}$, yield strength $\sigma_{\mathrm{ye}}$, and ultimate tensile strength $\sigma_{\mathrm{ue}}$ of the PNC have the following scaling 
relationships with respect to the constituent moduli $\left(E_{\mathrm{f}}\right.$ and $\left.E_{\mathrm{m}}\right)$, yield strength $\left(\sigma_{\mathrm{ym}}\right)$, and Poisson's ratios $\left(v_{\mathrm{f}}\right.$ and $\left.v_{\mathrm{m}}\right)$ and the volume fraction $\left(V_{\mathrm{f}}\right)$ and aspect ratio $(\rho)$ of the clay particles:

$$
\begin{aligned}
& \frac{E_{\mathrm{e}}}{E_{\mathrm{m}}}=f\left(\frac{E_{\mathrm{f}}}{E_{\mathrm{m}}}, \frac{\sigma_{\mathrm{ym}}}{E_{\mathrm{m}}}, v_{\mathrm{f}}, V_{\mathrm{m}}, V_{\mathrm{f}}, \rho\right) \\
& \frac{\sigma_{\mathrm{ye}}}{E_{\mathrm{m}}}=g\left(\frac{E_{\mathrm{f}}}{E_{\mathrm{m}}}, \frac{\sigma_{\mathrm{ym}}}{E_{\mathrm{m}}}, v_{\mathrm{f}}, V_{\mathrm{m}}, V_{\mathrm{f}}, \rho\right) \\
& \frac{\sigma_{\mathrm{ue}}}{E_{\mathrm{m}}}=h\left(\frac{E_{\mathrm{f}}}{E_{\mathrm{m}}}, \frac{\sigma_{\mathrm{ym}}}{E_{\mathrm{m}}}, v_{\mathrm{f}}, V_{\mathrm{m}}, V_{\mathrm{f}}, \rho\right)
\end{aligned}
$$

where $f, g$, and $h$ are, respectively, three unknown dimensionless functions with respect to the dimensionless ratios $E_{\mathrm{f}} / E_{\mathrm{m}}, \sigma_{\mathrm{ym}} / E_{\mathrm{m}}$, and dimensionless numbers $v_{\mathrm{f}}, v_{\mathrm{m}}, V_{\mathrm{f}}$ and $\rho$. In the above, subscript ' $\mathrm{e}$ ' denotes the effective properties of the PNC; subscript ' $\mathrm{f}$ ' denotes the properties of clay particles, and subscript ' $\mathrm{m}$ ' stands for the properties of the polymeric matrix. It needs to be mentioned that in reality, more parameters of a PNC system potentially govern the global mechanical properties of the PNC, such as the interfacial shear strength between the clay nano platelets and polymer resin, the morphology and orientation of the clay particles, among other. Yet, as a preliminary computational approach, the present study focuses only on the dominant parameters within the framework of classic mechanics of composite materials and computational micromechanics.

In typical PNCs, the clay particles are much stiffer and have much higher yield strength and ultimate tensile strength than those of the polymeric resins. Therefore, subjected to external loading, the clay particles are only in the range of elastic deformation till the catastrophic failure of the PNCs. As a reasonable deduction, the yield and tensile strengths of the clay particles can be ignored in the scaling relations (1-3). For the purpose of simplifying the upcoming discussions, the polymers in this study are assumed to be idealized elastoplastic solid, i.e., the yield strength $\sigma_{\text {ym }}$ equals to its ultimate tensile strength $\sigma_{\mathrm{um}}$. In addition, the yield strength of a PNC (2) $\sigma_{\mathrm{ye}}$ is physically less meaningful since in computational simulation such as FEA, localized tiny plastic deformation always exists in the polymeric matrix near the ends of the clay particles due to stress concentration at the clay particle edge/corner regions with abrupt geometrical change and depends also upon the extent of mesh refinement employed in FEA. However, the global mechanical properties of PNCs such as the tensile strength and fracture toughness are not so sensitive to such localized plastic deformation since the polymers are usually thermoplastic with excellent ductility and plastic deformation.

Besides, the transition region between the clay platelets and the polymeric matrix is not taken into account in the current study due to the following two considerations. First, physically, it is still rather difficult to identify the exact thickness of such interlayers based on available experimental data in the literature though many researchers have artificially introduced this ad hoc region to show the size effect in the mechanical properties of nanocomposites. Second, some recent simulations such as those provided by Dong and Bhattacharyya [21] showed less impact of the interlayers on the mechanical properties of PNCs, which might be related to the small volume fraction of the interlayers and the clay particles in the model. Thus, introduction of the interlayers to modeling the mechanical properties of PNCs is still a controversial topic and needs more in-depth experimental evidence and physical justification. Thus, in the present study, we mainly focused on the scaling properties of the effective modulus (1) and the ultimate tensile strength (2) of the PNCs with respect to the two major processing parameters: the clay-particle volume fraction $V_{\mathrm{f}}$ and geometrical aspect ratio $\rho$. A detailed 2D nonlinear FEA was conducted for this purpose.

\subsection{FEM-Based Computational Micromechanics Simulation}

In processing a PNC, clay particles can be potentially distributed in the polymeric matrix in three different extents, i.e., the conventional micron level particles, intercalated particle and exfoliated nano 
platelets, as illustrated in Figure 1. The latter two are the general states to achieve PNCs with preferable mechanical properties.

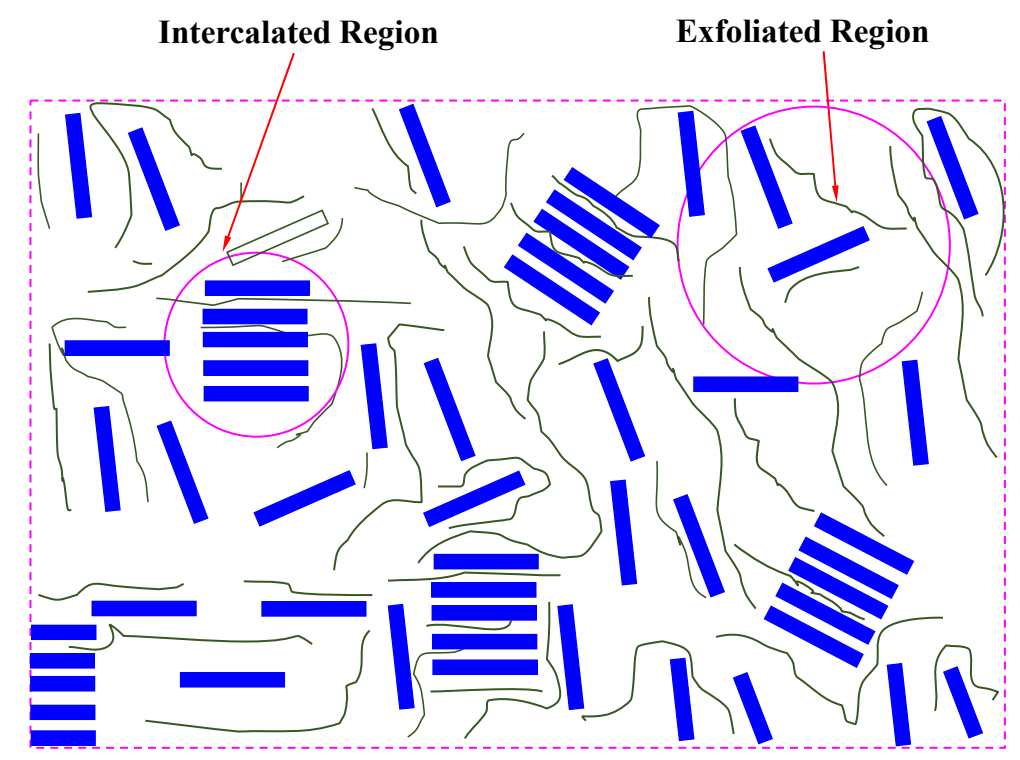

Figure 1. Schematic of intercalated and exfoliated clay particles in a polymeric matrix.

To investigate the scaling properties of PNCs with varying clay-particle volume fraction $V_{\mathrm{f}}$ and aspect ratio $\rho$, we considered the typical stacking and staggering distributions of identical clay particles in the polymeric matrix. The RVEs to be extracted for FEM simulations are based on the periodical and symmetrical conditions of the PNCs subjected to axial tension along the particle axis, as shown in Figures 2 and 3, respectively.
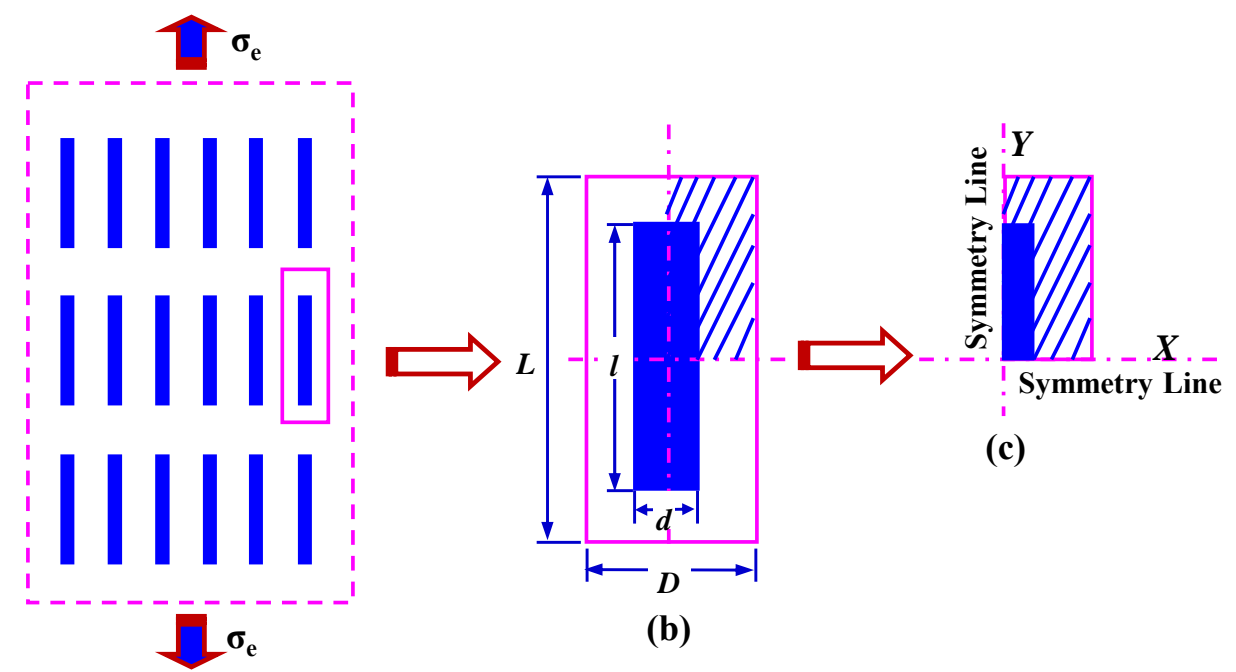

(a)

(b)

Figure 2. Representative volume element (RVE) of the stack model used for the present computational scaling analysis. (a) Idealized identical stacking clay platelets; (b) a typical RVE; and (c) a quarter RVE for efficient simulation. 


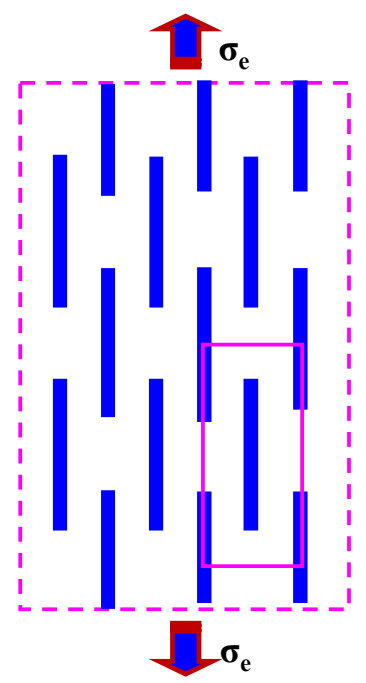

(a)

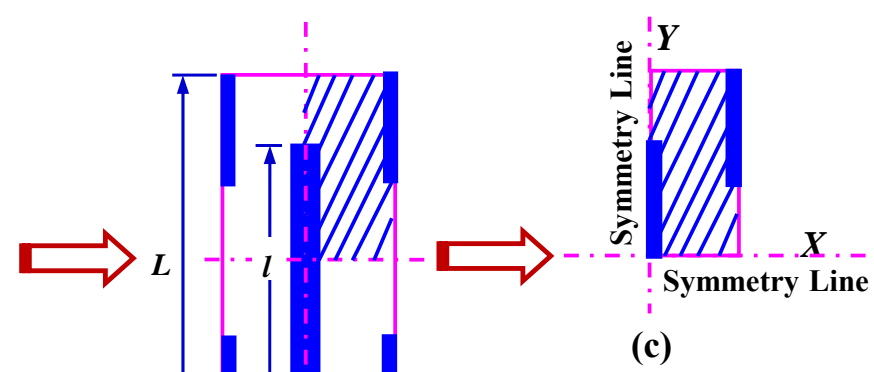

(b)

Figure 3. Representative volume element (RVE) of the stagger model used for the present computational scaling analysis. (a) Idealized identical staggering clay platelets; (b) a typical RVE; and (c) a quarter RVE for efficient simulation.

During the computational simulation, a wide range of clay-particle aspect ratios $(\rho=1,2,5,10,20$, 50 and 100$)$ and volume fractions $\left(V_{\mathrm{f}}=0.5 \%, 1 \%, 2 \%, 5 \%, 7.5 \%\right.$, and $\left.10 \%\right)$ were utilized. Additionally, the stack and stagger models with the filler volume fraction $15 \%$ and aspect ratio 100 have been also considered for the purpose of comparative study, to examine their effects on the ultimate tensile strength and effective modulus of the resulting PNCs. The aspect ratio $\rho$ and volume fraction $V_{\mathrm{f}}$ can be obtained by adjusting the RVE dimensions: $L, D, l$, and $d$ as

$$
\rho=\frac{l}{d} \text { and } V_{\mathrm{f}}=\frac{l d}{L D} .
$$

The mechanical properties of the polymeric matrix and clay particles were selected close to those reported in the literature $[4,6]$. The polymeric matrix was modeled as an idealized elastoplastic solid with the yield strength $\sigma_{\mathrm{ym}}=79.0 \mathrm{MPa}$, Young's modulus $E_{\mathrm{m}}=2.75 \mathrm{GPa}$, and Poisson's ratio $v_{\mathrm{m}}=0.41$; the clay particles were modeled as linearly elastic solid with Young's modulus $E_{\mathrm{f}}=178 \mathrm{GPa}$ and Poisson's ratio $v_{\mathrm{f}}=0.28$.

2D nonlinear FEA was conducted to determine the entire stress field of the quarter RVEs (see Figures 2c and 3c) of the PNCs subjected to axial stretching. During the process, a plane-strain 4-node 182 element offered by ANSYS ${ }^{\mathrm{TM}}$ was adopted; symmetrical boundary conditions were enforced at the horizontal and vertical symmetric axes; forced displacement constraints were triggered to maintain the constant horizontal displacements at the right vertical edges; no debonding was assumed between the clay particle and the matrix. The nonlinear FEA was executed under the condition of small displacement; the Newton-Raphson algorithm was selected for the nonlinear numerical iterations. The convergence criterion, equation solver, and nonlinear options were set as program defaults. After each simulation, the nodal forces at the top edge were extracted and recorded at several sampling displacements (i.e., effective tensile strains) until the effective strain equaled three times the yield strain of the polymeric matrix; the corresponding effective tensile stresses were calculated manually by dividing the sum of the edge nodal forces by the width of the RVE. The numerical experiments showed that the above nonlinear FEA can give reliable effective stress-stain diagrams of the PNCs at varying clay-particle aspect ratio $\rho$ and volume fraction $V_{\mathrm{f}}$ within this consideration. Detailed numerical results will be discussed in Section 3. 


\section{Results and Discussion}

In the present computational micromechanics scaling study, FEM-based nonlinear analysis was conducted to cover a wide range of clay-particle aspect ratios $(\rho=1,2,5,10,20,50$ and 100) and volume fractions $\left(V_{\mathrm{f}}=0.5 \%, 1 \%, 2 \%, 5 \%, 7.5 \%\right.$, and $\left.10 \%\right)$ for both the stack and stagger models. The effective moduli predicted by the two idealized clay-particle arrangements (i.e., stacking and staggering) are nearly the same, especially at the low volume fraction of dilute clay particles in the polymeric matrix. This is the trivial conclusion that can be easily drawn in linearly elastic micromechanics as the effective moduli of PNCs of aligned clay particles are independent of either stacking or staggering arrangement in the dilute clay particle state, and the small variation between the two models are possibly induced by the difference of localized plastic deformations and a small deviation of the clay particle interaction in the two micromechanics models. Compared to the effective moduli, the effective ultimate tensile strengths of the PNCs have a little noticeable variation between the stack and stagger models. However, the general varying trends of the effective moduli and ultimate tensile strengths based on the two computational micromechanics models are consistent and will be discussed further. Hereafter, only the results based on the stack model are shown though a comparative study will also be presented later on. In the case of the stack model, variation of the effective elastic modulus $E_{\mathrm{e}}$ and effective ultimate tensile strength $\sigma_{\text {ue }}$ with the varying clay-particle volume fraction $V_{\mathrm{f}}$ at several aspect ratios are shown in Figures 4 and 5, respectively. During the data reduction process, the effective moduli in Figure 4 were extracted from the slope of the initial linear elastic region of the effective tensile stress-strain diagrams $\left(\sigma_{\mathrm{e}}-\varepsilon_{\mathrm{e}}\right)$ of all the computational cases as shown in Figures 6-11. These results indicate a very good linear relationship between the effective tensile stress $\sigma_{\mathrm{e}}$ and the effective tensile strain $\varepsilon_{\mathrm{e}}$ as expected. From Figures 6-11, it can be observed that when the value of effective tensile strain $\varepsilon_{\mathrm{e}}$ is low, the PNCs behave as linearly elastic solids until the effective tensile strain $\varepsilon_{\mathrm{e}}$ is close to the yield strain of the polymeric matrix. The effective elastic moduli $E_{\mathrm{e}}$ extracted from the present numerical experiments (see Figure 4) are close to those available in the literature [11,12]. In addition, it can also be found from Figures 6-11 that the PNCs exhibit slightly softening behavior prior to the arrival of the yield strain of the polymeric matrix. This phenomenon can be attributed to the localized plastic deformation near the ends of clay particles where stress concentration exists due to the sharp edges/corners of the clay particles. In reality, at the nanoscale, the physical/chemical properties of the polymer chains close to the clay platelets are much more complicated than the present material model and are also noticeably different from the polymers in bulk state. Such unusual properties of the interfacial polymers at the nanoscale are beyond the scope of this computational study and are still under intensive investigation.

In addition, at the low clay-particle volume fraction $\left(V_{\mathrm{f}}<2 \%\right)$ or low aspect ratio $(\rho=1,2$, 5 , and 10) based on the present computational study, no obvious improvement of the effective ultimate tensile strength $\sigma_{\text {ult }}$ is detected as shown in Figure 5. This characteristic can be also examined from the $\sigma_{\mathrm{e}}-\varepsilon_{\mathrm{e}}$ diagrams given in Figures 6-11. Such an observation is also qualitatively in agreement with the recent experimental results of the mechanical properties of PNCs as reported in the literature $[4,6]$. In these cases, the values of effective ultimate tensile strength $\sigma_{\text {ult }}$ are still dominated by the yield strength of the polymeric matrix; the contribution of the load-carrying capacity of clay particles to the nanocomposites is negligible. Such an observation can be understood as follows. Physically, different from the effective stiffness which is governed by the averaging effect of the mechanical properties of the constituents forming the PNCs, the dilute clay nanoparticles in the polymer matrix are unable to form load-transferring bridges and therefore unable to noticeably strengthen the resulting PNCs. Therefore, dilute clay nanoparticles at low volume concentration have less impact on the hardening of the polymeric matrix. However, the effective ultimate tensile strength $\sigma_{\mathrm{ult}}$ of the PNCs is significantly enhanced in the cases of $V_{\mathrm{f}}=5 \%$ and $10 \%$ and $\rho=50$ as shown in Figures 10 and 11. In the case of $V_{\mathrm{f}}=5 \%$ and $\rho=20$ (Figure 10), the improvement of the effective ultimate tensile strength $\sigma_{\text {ult }}$ of the PNC reaches $\sim 60 \%$ on the basis of the yield strength of the virgin polymeric matrix; this increment is close to those achieved experimentally in Nylon-6 
and silicone rubber nanocomposites reinforced with montmorillonite clay particles [4]. Moreover, in the case of $V_{\mathrm{f}}=10 \%$ and $\rho=50$, the present simulation predicts the increment of the effective ultimate tensile strength $\sigma_{\text {ult }}$ of the PNCs up to $\sim 150 \%$. This value is much higher than the ones reported in the literature. In realistic PNCs, the polymer/clay interphase, interfacial shear failure (sliding/pull-out failure), filler waviness, and orientation will noticeably decrease this value. In the above two cases, the clay particles with an aspect ratio $\rho$ higher than 10 apparently increase the load-carrying capacity of the resulting PNCs.

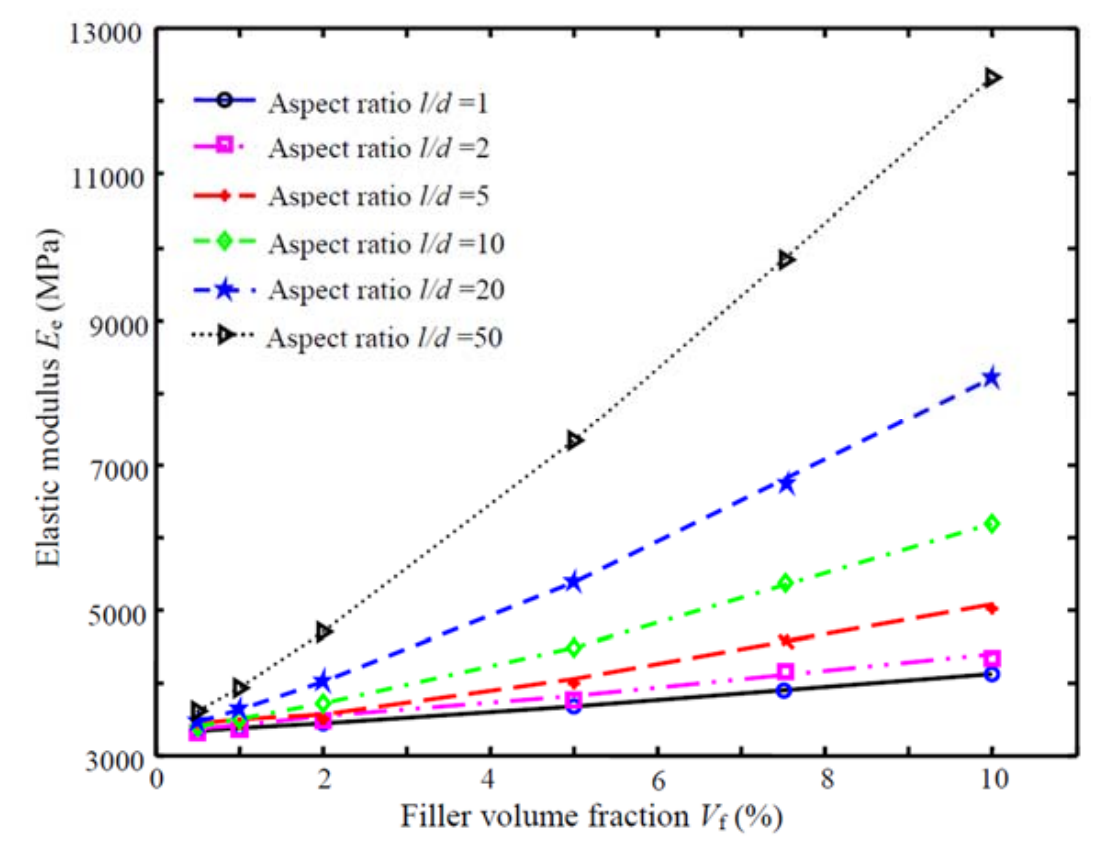

Figure 4. Variation of the effective elastic modulus $E_{\mathrm{e}}$ with the filler volume fraction $V_{\mathrm{f}}$.

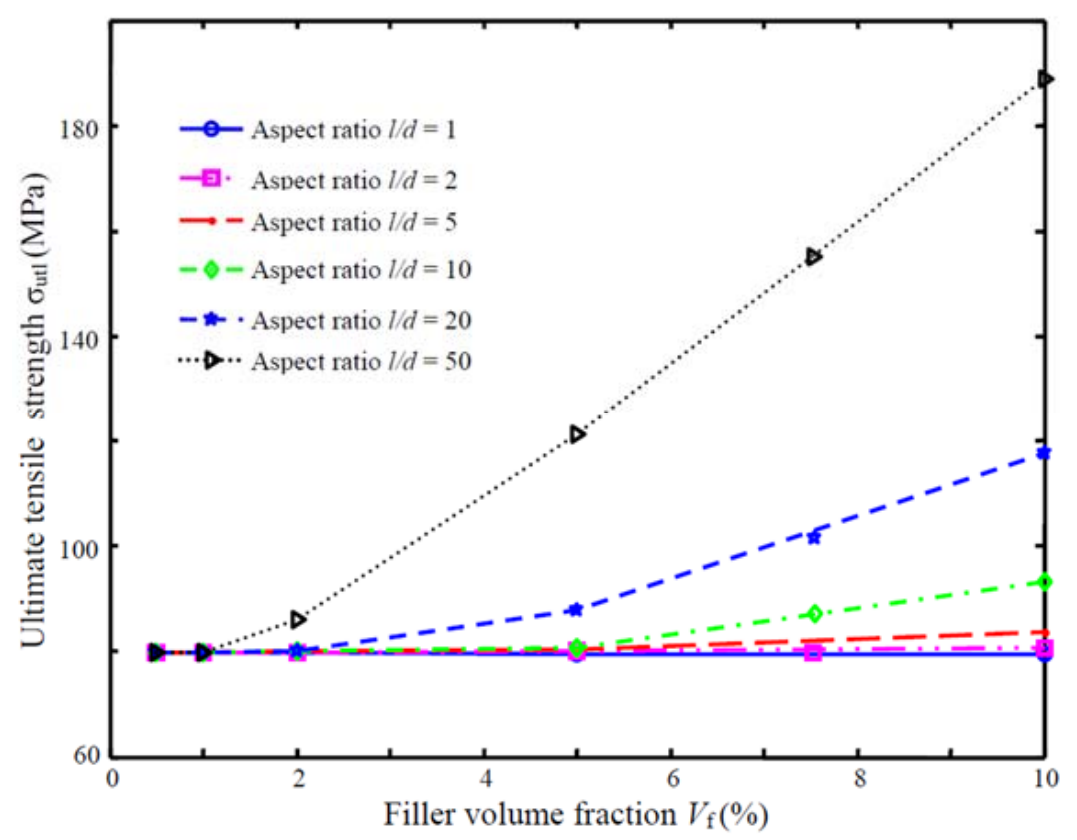

Figure 5. Variation of the effective ultimate tensile strength $\sigma_{\mathrm{utl}}$ with the filler volume fraction $V_{\mathrm{f}}$. 


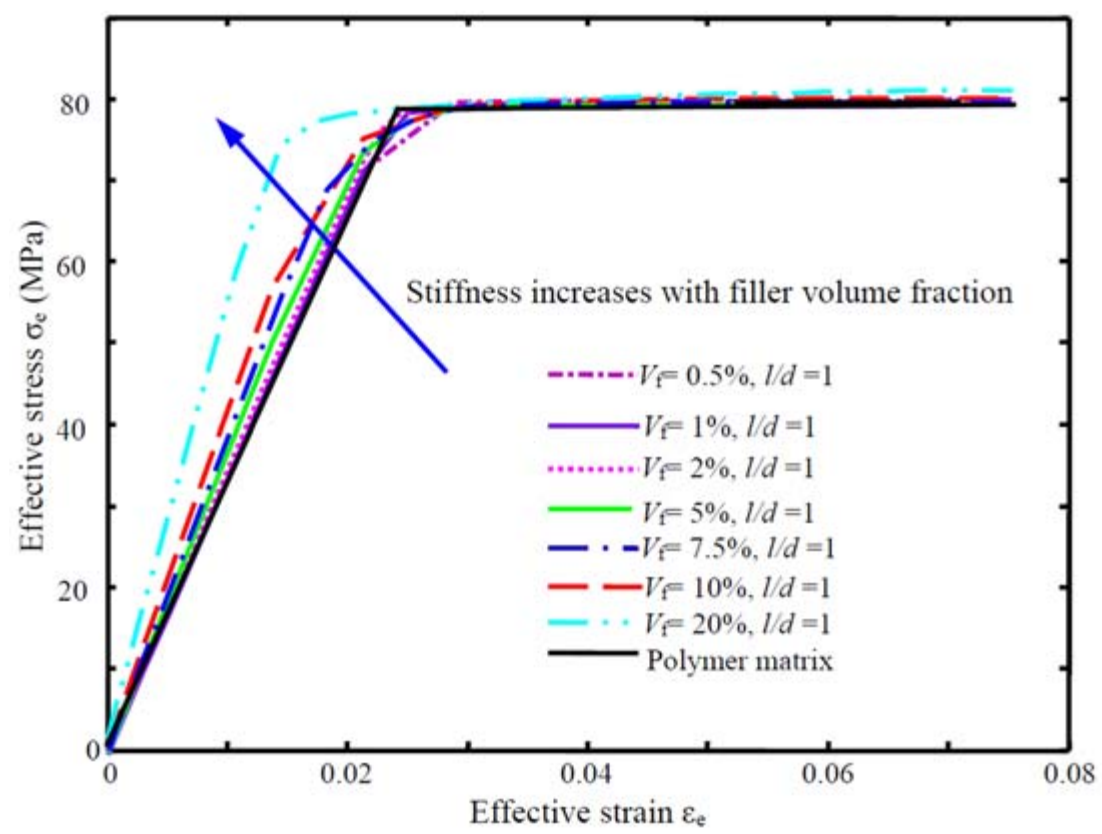

Figure 6. Effective stress-strain $\sigma_{\mathrm{e}}-\varepsilon_{\mathrm{e}}$ diagram of the PNCs with the clay-particle aspect ratio $\rho=1$ (plane-strain, case I).

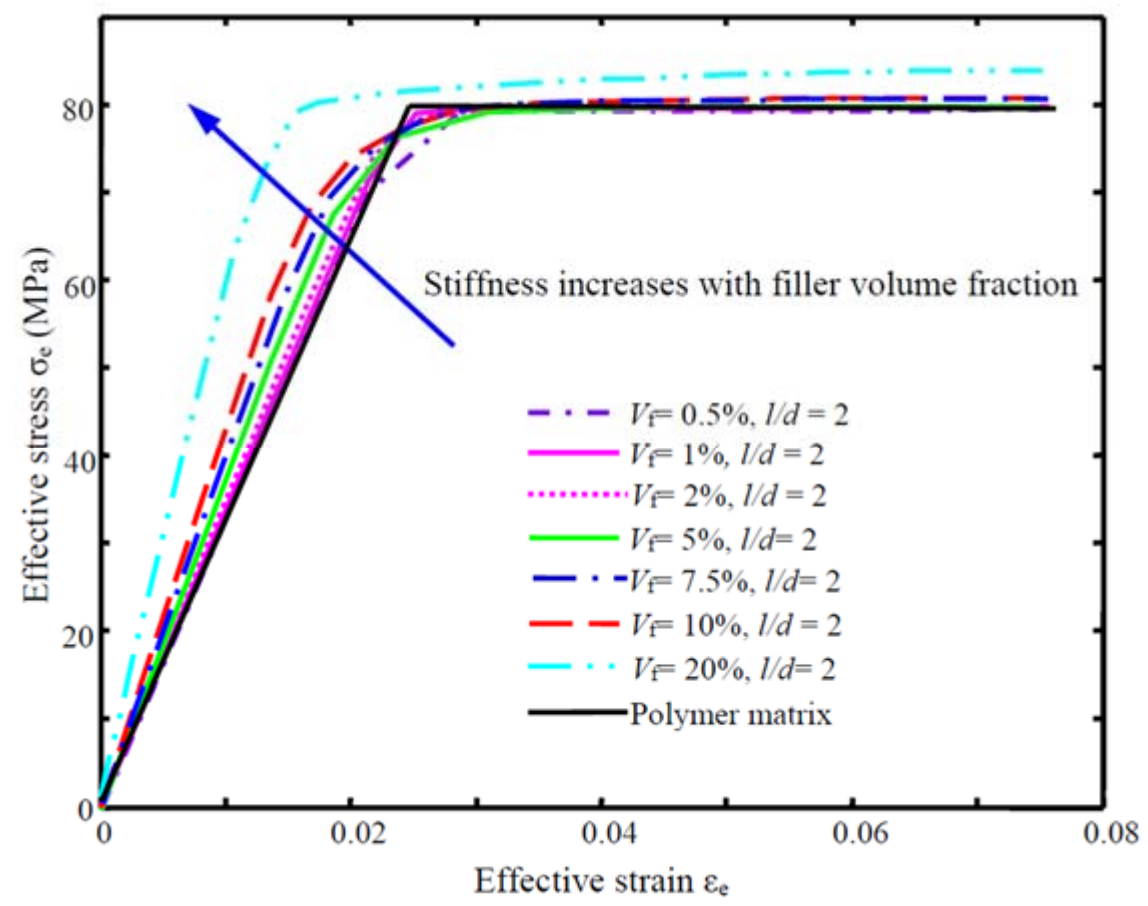

Figure 7. Effective stress-strain diagram $\sigma_{\mathrm{e}}-\varepsilon_{\mathrm{e}}$ of the PNCs with the clay-particle aspect ratio $\rho=2$ (plane-strain, case I). 


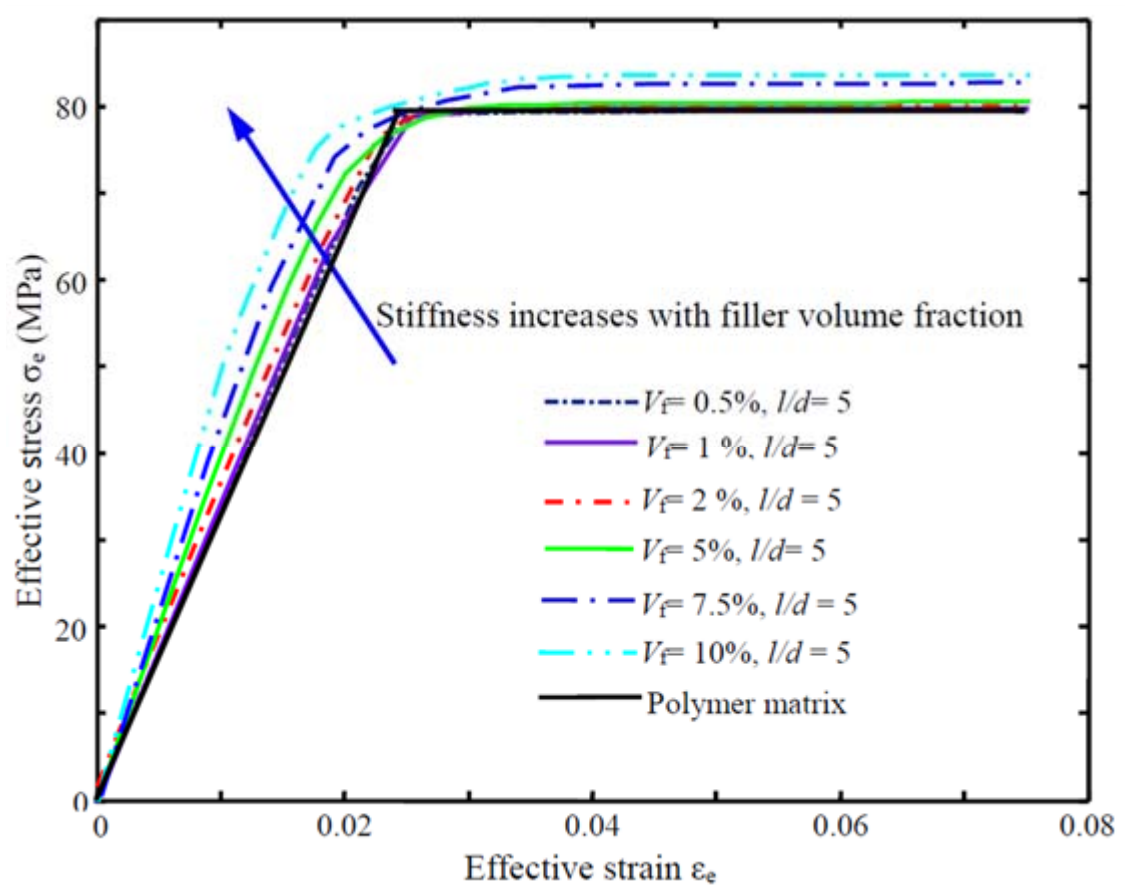

Figure 8. Effective stress-strain diagram $\sigma_{\mathrm{e}}-\varepsilon_{\mathrm{e}}$ of the PNCs with the clay-particle aspect ratio $\rho=5$ (plane-strain, case I).

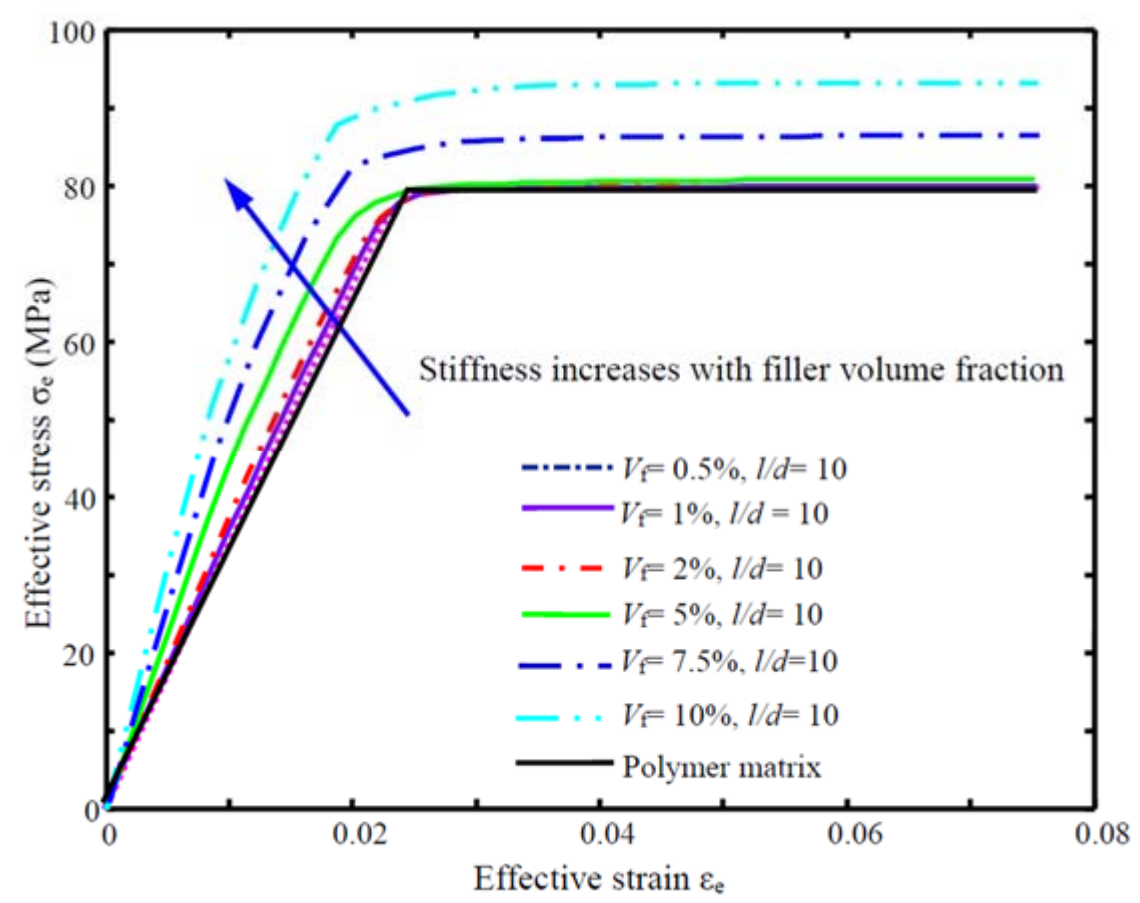

Figure 9. Effective stress-strain diagram $\sigma_{\mathrm{e}}-\varepsilon_{\mathrm{e}}$ of the PNCs with the clay-particle aspect ratio $\rho=10$ (plane-stain, case I). 


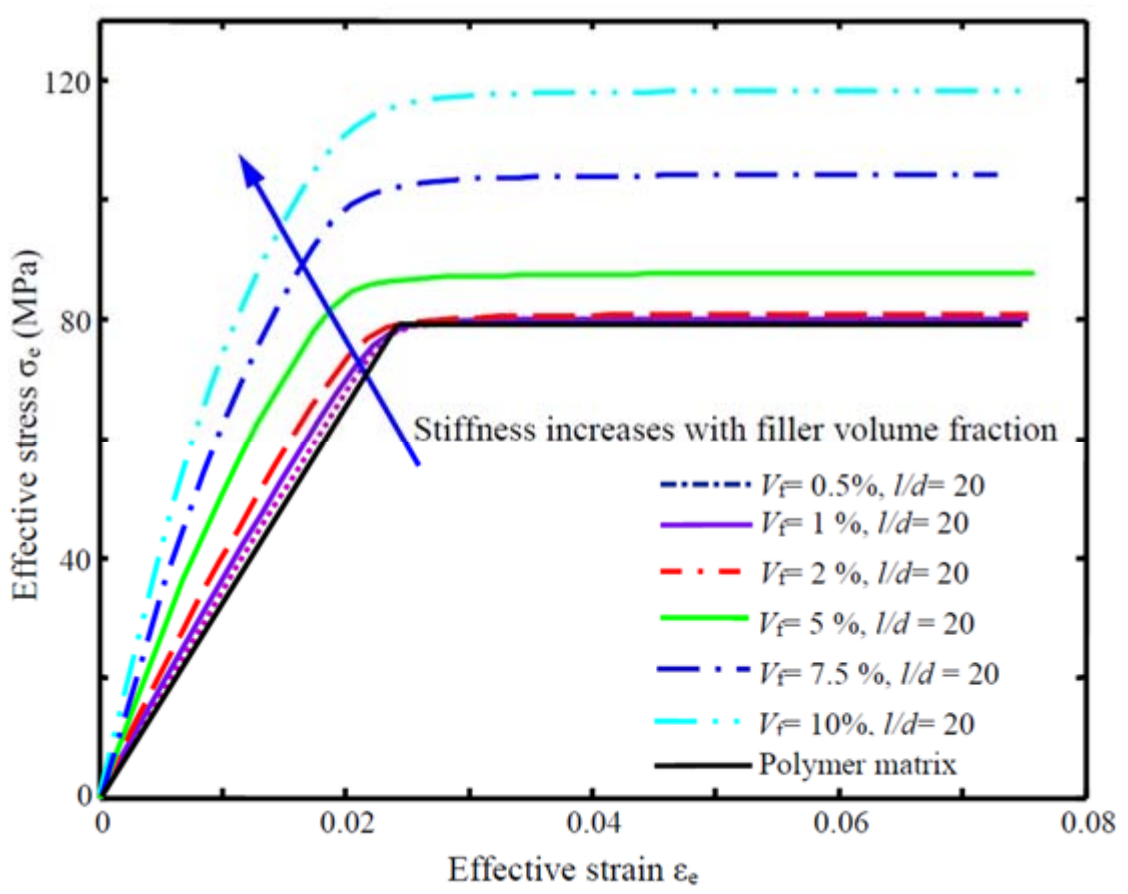

Figure 10. Effective stress-strain diagram $\sigma_{\mathrm{e}}-\varepsilon_{\mathrm{e}}$ of the PNCs with the clay-particle aspect ratio $\rho=20$ (plane-strain, case I).

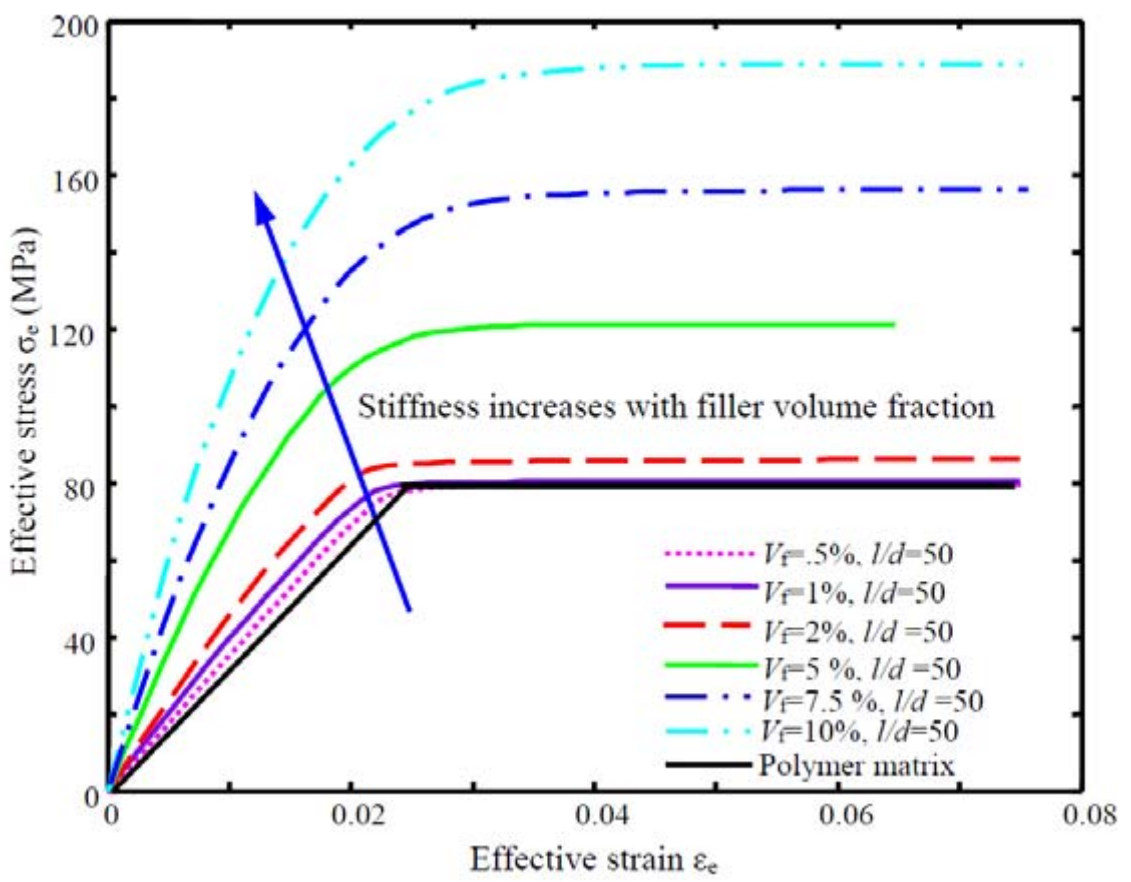

Figure 11. Effective stress-strain diagram $\sigma_{\mathrm{e}}-\varepsilon_{\mathrm{e}}$ of the PNCs with the clay-particle aspect ratio $\rho=50$ (plane-strain, case I).

Furthermore, the FEA results indicate that at the low particle aspect ratios (e.g., $\rho=1,2,5$ and 10), the effective ultimate tensile strengths $\sigma_{\text {ult }}$ of the PNCs based on the two models are nearly independent of the clay volume fraction $V_{\mathrm{f}}$ up to $5 \%$, i.e., the polymeric matrix governs the tensile strength of the PNCs. This observed phenomenon is due to the fact that the relatively dilute clay nanoparticles in polymeric matrix are unable to form load-transferring bridges at the low aspect ratios 
of the clay particles to strengthen the resulting PNCs. Besides, by examining the results obtained in the cases of the filler aspect ratio $\rho$ at 10, 20, 50 and 100 and the filler volume fraction $V_{\mathrm{f}}$ at $0.5 \%, 1 \%$, $2 \%, 5 \%, 10 \%$ and $15 \%$, respectively, it can be found from Figures $12-15$ that when the effective tensile strain $\varepsilon_{\mathrm{e}}$ is low, the PNCs behave as linearly elastic solids until the effective strain $\varepsilon_{\mathrm{e}}$ close to the yield strain of the polymeric matrix and the stack and stagger models both predict very similar effective tensile moduli. In addition, the PNCs exert slightly softening behavior prior to the arrival of the yield strain of the polymeric matrix due to stress-concentration induced matrix (resin) yielding near the filler ends. In addition, the percent increase of the effective modulus $E_{\mathrm{e}}$ for both the stack and stagger models as shown in Figure 16 indicates that for all the aspect ratios, the computational predictions based on the stack and stagger models are close, especially at the dilute clay particle state, as confirmed by linearly elastic micromechanics. Yet, a small variation of the effective tensile modulus between the stack and stagger models can be detected, which is due to the variation of clay particle interference between the two models. Moreover, Figure 17 shows the comparative effective ultimate tensile strength $\sigma_{\text {ult }}$ of PNCs predicted by the stack and stagger models. It can be observed that the trend of ultimate tensile strength $\sigma_{\text {ult }}$ increase is the same for the two models; however, the deviation of the predicted effective tensile strength increases with increasing aspect ratio $\rho$ for the two models, and the stagger model predicts relatively lower values of the effective tensile strength than the stack model does for all the computational cases under the present consideration. By comparing the clay-particle arrangements in the two models, it can be concluded that the clay nanoparticles in stagger model are arranged more uniform than those in the stack model at a given filler volume fraction. Thus, subjected to the same effective tensile strain, the stack model generated more uneven stress field, or a higher stress gradient, than the stagger model, i.e., a higher ultimate tensile strength by the stack model. Such an observation also indicates that the ultimate tensile strength is more sensitive to the clay nanoparticle arrangement in the model, which can be useful to process strength-controllable PNCs.

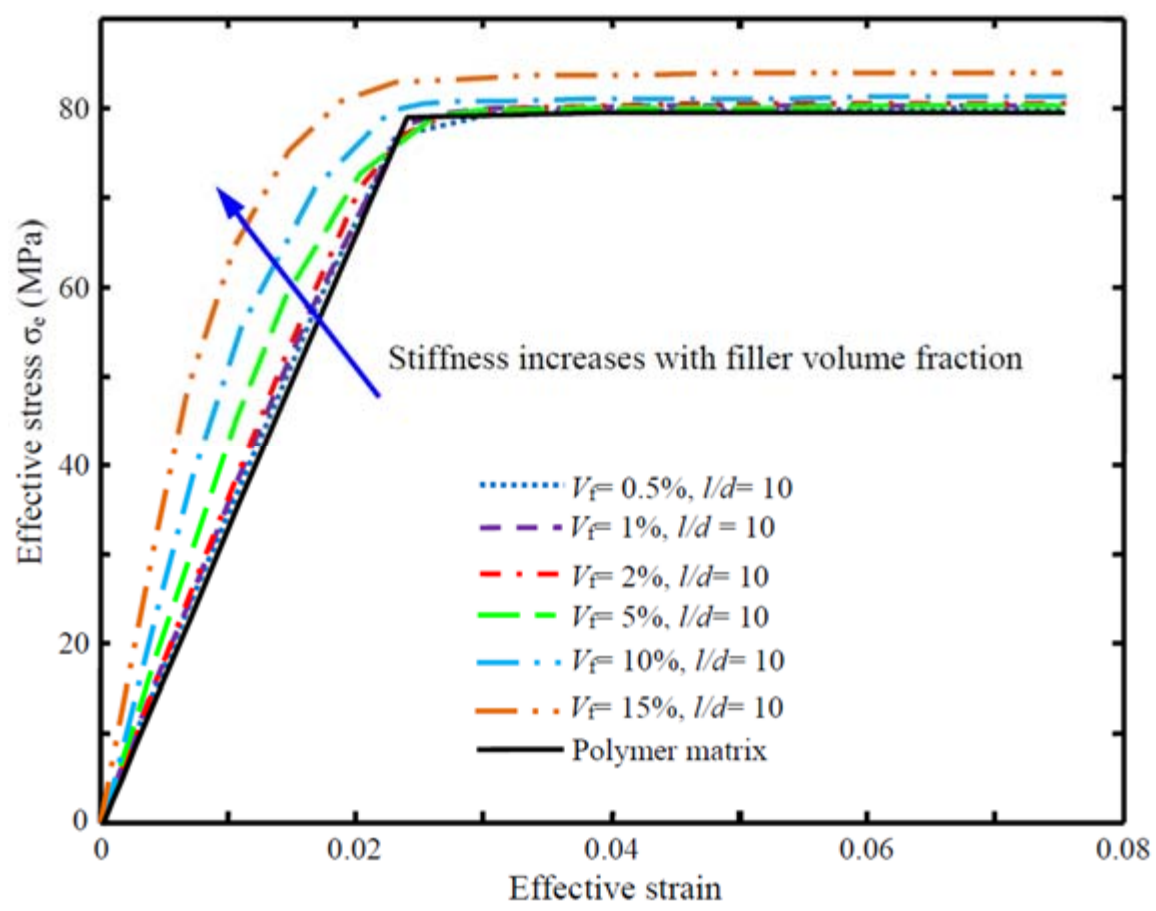

Figure 12. Effective stress-strain diagram $\sigma_{\mathrm{e}}-\varepsilon_{\mathrm{e}}$ of the PNCs with the clay-particle aspect ratio $\rho=10$ (plane-stain, case II). 


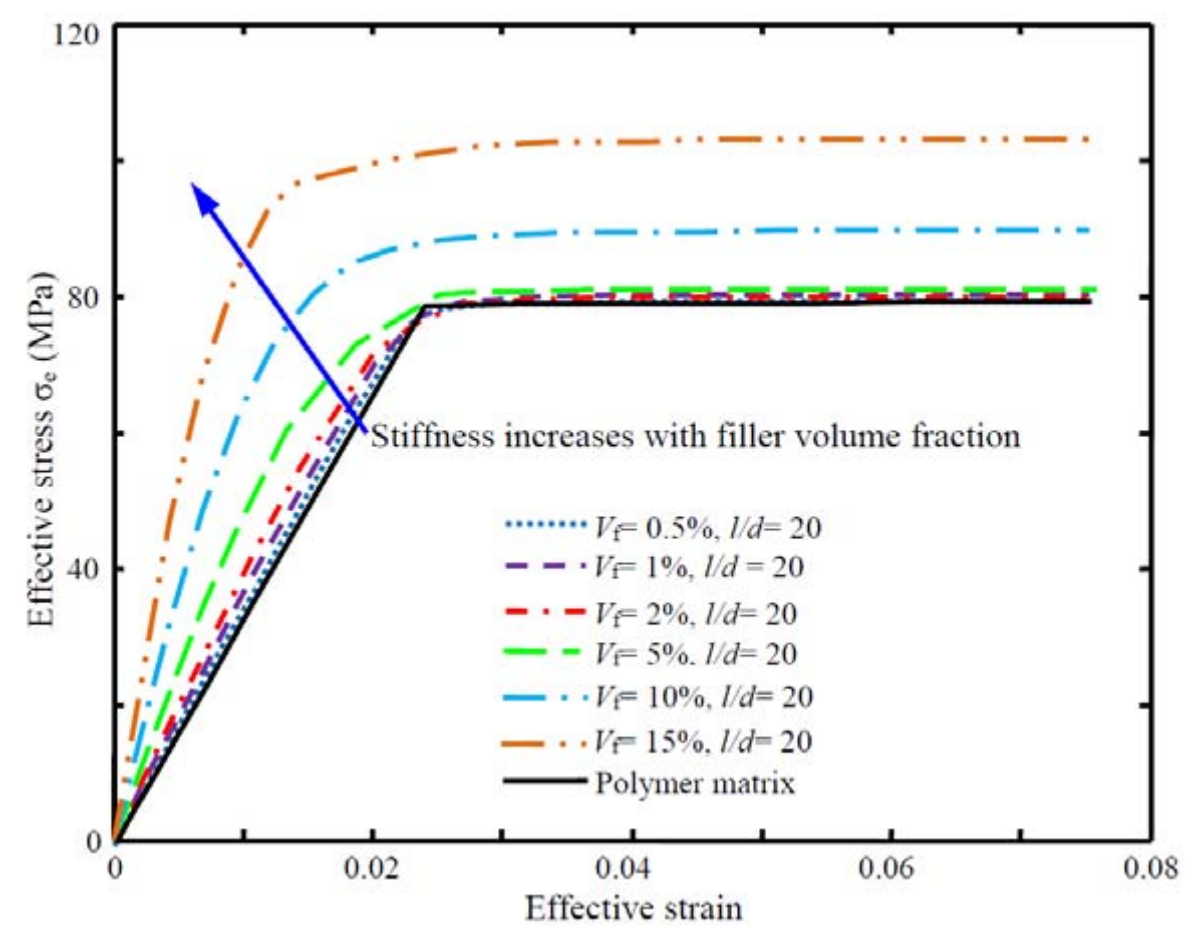

Figure 13. Effective stress-strain diagram $\sigma_{\mathrm{e}}-\varepsilon_{\mathrm{e}}$ of the PNCs with the clay-particle aspect ratio $\rho=20$ (plane-strain; case II).

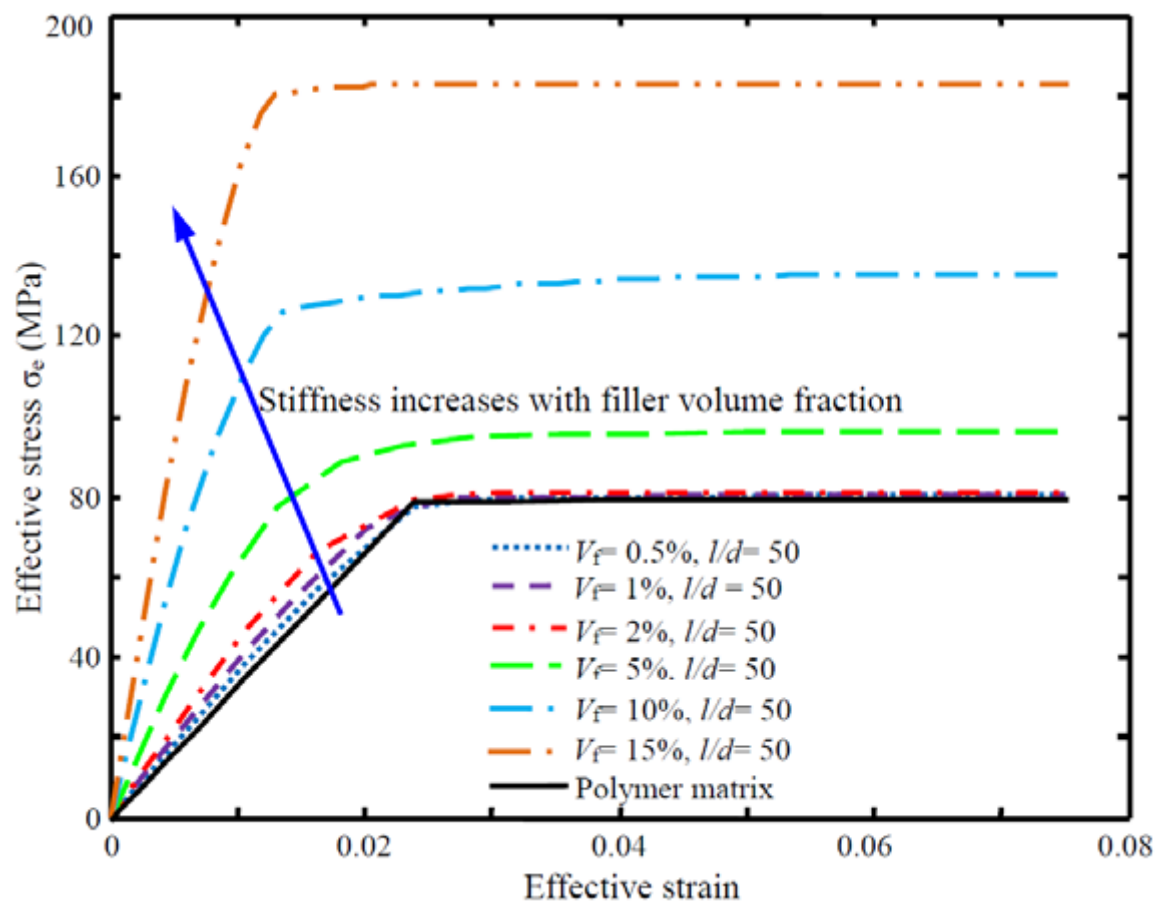

Figure 14. Effective stress-strain diagram $\sigma_{\mathrm{e}}-\varepsilon_{\mathrm{e}}$ of the PNCs with the clay-particle aspect ratio $\rho=50$ (plane-strain, case II). 


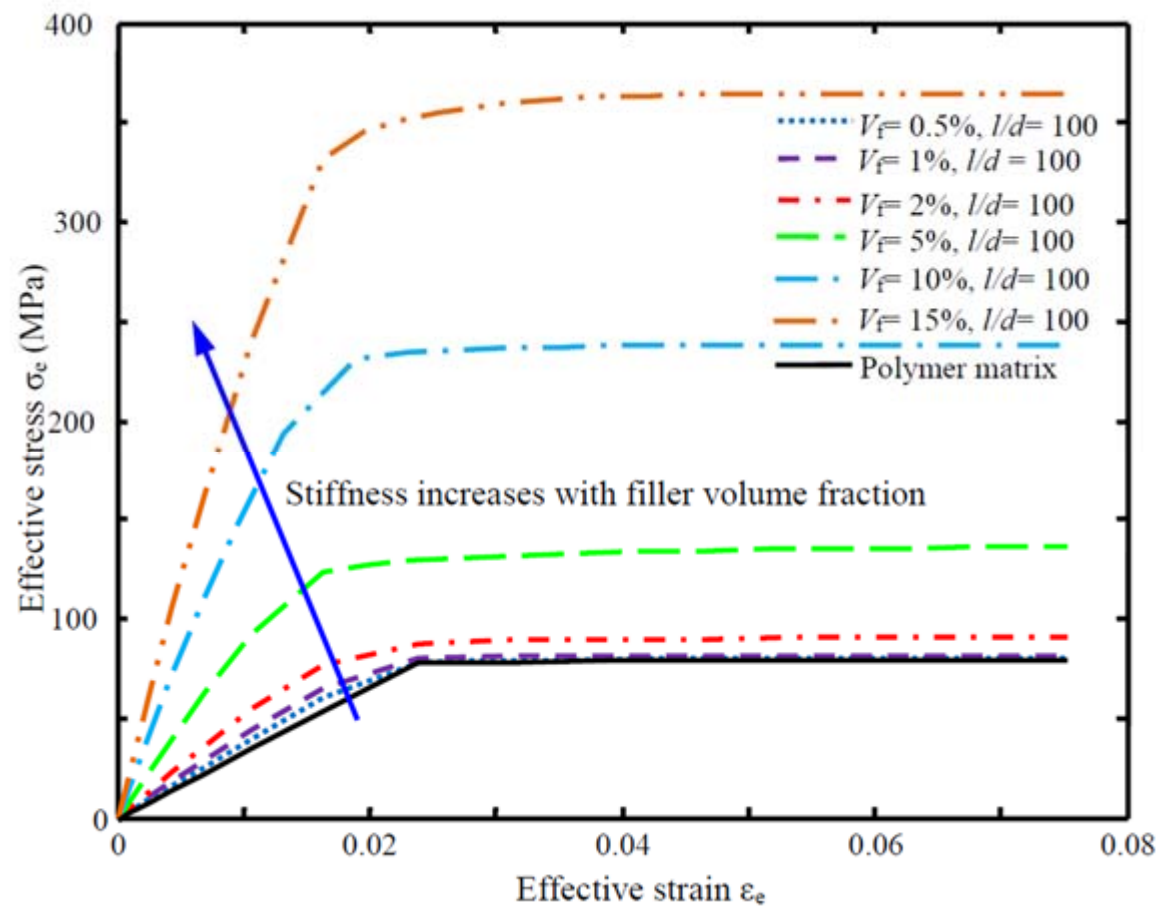

Figure 15. Effective stress-strain diagram $\sigma_{\mathrm{e}}-\varepsilon_{\mathrm{e}}$ of the PNCs with the clay-particle aspect ratio $\rho=100$ (plane-strain, case II).

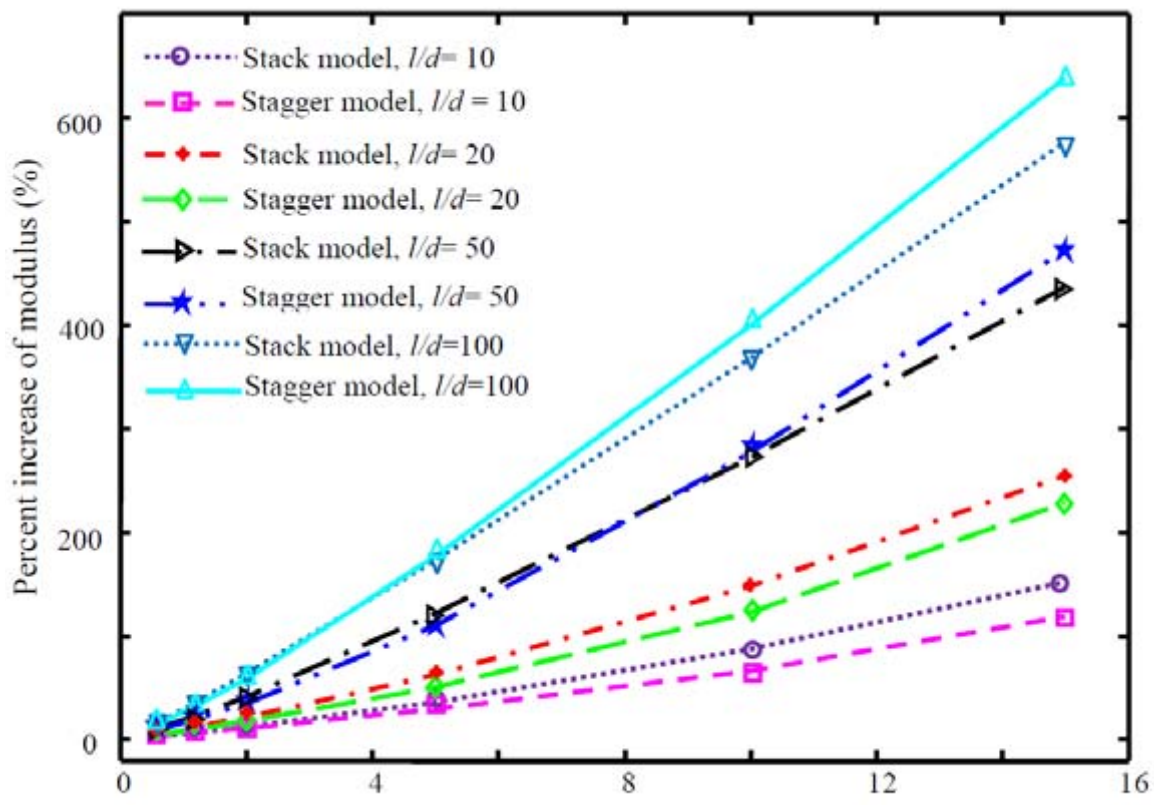

Filler volume fraction $V_{\mathrm{f}}(\%)$

Figure 16. Variation of the percent increase of effective modulus (compared to matrix) with the filler volume fraction $V_{\mathrm{f}}$ for different clay-particle aspect ratios $\rho=10,20,50$ and 100. 


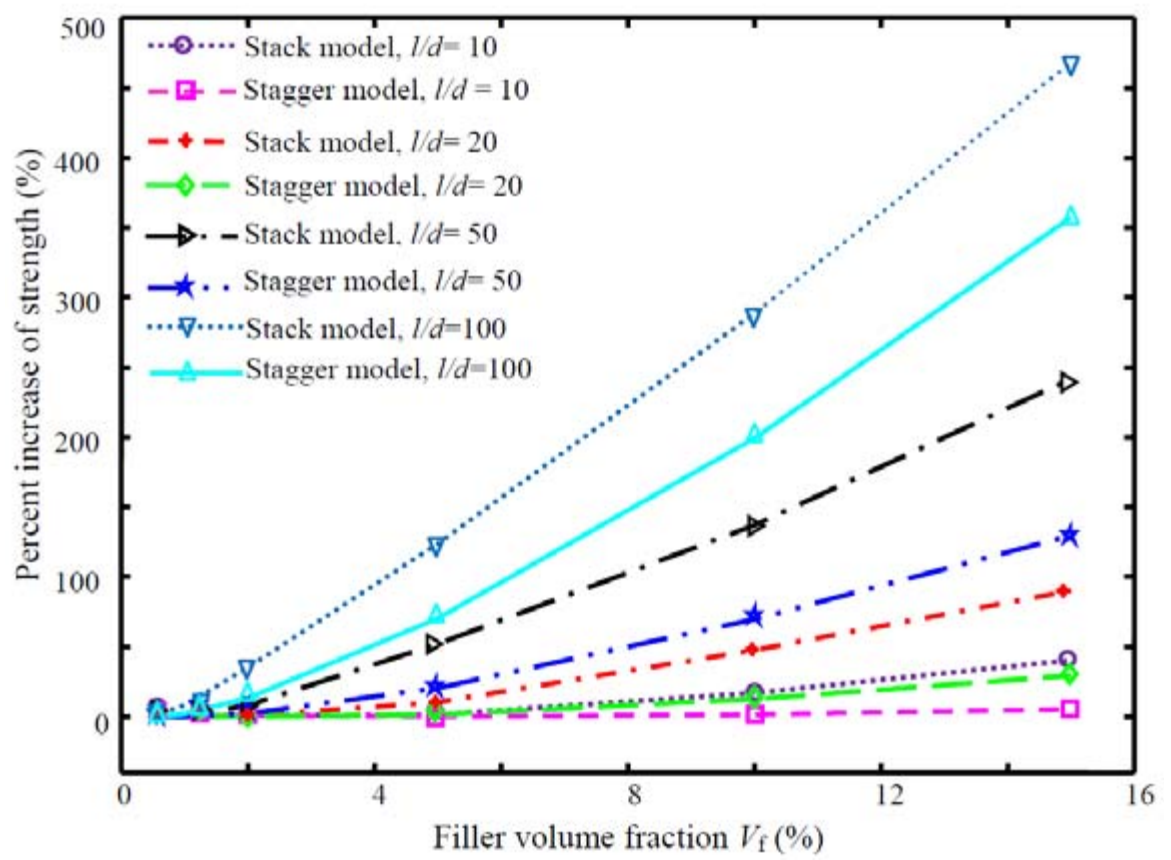

Figure 17. Variation of the percent increase of tensile strength (compared to matrix) with the filler volume fraction $V_{\mathrm{f}}$ for different clay-particle aspect ratios $\rho=10,20,50$ and 100.

It needs to be mentioned that in reality, the aspect ratio of fully exfoliated clay platelets is much larger than the values studied in this work. Yet, not well aligned clay platelets in polymeric matrices will substantially weaken the potential improvement of the effective tensile stiffness $E_{\mathrm{e}}$ and ultimate tensile strength $\sigma_{\text {eu }}$ of the realistic PNCs in a specific orientation. This implies the tailorability of the effective tensile moduli and ultimate tensile strength of PNCs via optimal and controlled clay-particle exfoliation and orientation. To date, efficient processing techniques are still desired to effectively achieve controlled platelet orientation in polymeric matrix. Though the present study was based on the simple stack and stagger models, the computational results provide the insight to understand the scaling mechanical properties of PNCs. Such computational models can be further enhanced to involve other processing parameters such as platelet orientation, waviness and extent of intercalated clay particles for predicting the mechanical behavior of PNCs in controlled fabrication.

\section{Concluding Remarks}

Computational stack and stagger micromechanics models were formulated and integrated into 2D FEA of PNCs for examining the effects of two key processing parameters (i.e., the clay-particle volume fraction $V_{\mathrm{f}}$ and aspect ratio $\rho$ ) on the mechanical behavior of PNCs. The present computational scaling studies have shown that the clay-particle aspect ratio $\rho$ plays a crucial role in enhancing the ultimate tensile strength of PNCs. Specifically, the aspect ratio $\rho>50$ is preferable to achieve significantly improved mechanical properties of PNCs. The stack and stagger models predicted the close effective moduli and slightly different ultimate tensile strengths due to the model sensitivity in the case of plastic failure of the PNCs. As a matter of fact, completely stacked and staggered clay platelets are two limiting aligned cases (best alignment) of PNCs reinforced with fully exfoliated clay nanoparticles, which predict the effective mechanical properties that can be considered as the theoretical bounds useful to guide experimental studies. The present study was based on classic computational micromechanics, the conclusions drawn from this study can also hold for other conventional composites reinforced with particles and short fibers. This study can be further extended to integrate additional processing and material parameters of PNCs. Finally, the present computational approach offers a technical tool for 
the possibility of efficient computer-aided nanocomposite design for targeted mechanical properties and quality-controllable nanocomposite manufacturing.

Acknowledgments: Partial support of the research by the NASA EPSCoR (NASA Grant \#NNX07AK81A, seed grant: 43500-2490-FAR018640), NDSU Development Foundation (Grant No.: 43500-2490-FAR0017475), and NDSU Faculty Research Initial Grant is gratefully appreciated.

Author Contributions: Xiang-Fa Wu conceived and designed the computational study; Arifur Rahman conducted all the FEM simulations and figure plotting; Xiang-Fa Wu and Arifur Rahman analyzed the data; Xiang-Fa Wu wrote the paper.

Conflicts of Interest: The authors declare no conflict of interest.

\section{References}

1. Fukushima, Y.; Okada, A.; Kawasumi, M.; Kurauchi, T.; Kamigaito, O. Swelling behavior of montmorillonite by poly-6-amide. Clay Miner. 1998, 23, 27-34. [CrossRef]

2. Usuki, A.; Kojima, Y.; Kawasumi, M.; Okada, A.; Fukushima, Y.; Kurauchi, T.; Kamigaito, O. Synthesis of nylon-6-clay hybrid. J. Mater. Res. 1993, 8, 1179-1183. [CrossRef]

3. Kojima, Y.; Usuki, A.; Kawasumi, M.; Okada, A.; Kurauchi, T.; Kamigaito, O. Synthesis of nylon-6-clay hybrid by montmorillonite intercalated with E-caprolactam. J. Polym. Sci. Part A Polym. Chem. 1993, 31, 983-986. [CrossRef]

4. Alexandre, M.; Dubois, P. Polymer-layered silicate nanocomposites: Preparation, properties and uses of a new class of materials. Mater. Sci. Eng. R 2000, 28, 1-63. [CrossRef]

5. Ray, S.S.; Okamoto, M. Polymer/layered silicate nanocomposites: A review from preparation to processing. Prog. Polym. Sci. 2003, 28, 1359-1641.

6. Tjong, S.C. Structural and mechanical properties of polymer nanocomposites. Mater. Sci. Eng. R 2006, 53, 73-197. [CrossRef]

7. Balazs, A.C.; Emrick, T.; Russell, T.P. Nanoparticle polymer composites: Where two small worlds meet. Science 2006, 314, 1107-1110. [CrossRef] [PubMed]

8. Schadler, L.S.; Brinson, L.C.; Sawyer, W.G. Polymer nanocomposites: A small part of the story. JOM 2007, 59, 53-60. [CrossRef]

9. Paul, D.R.; Robeson, L.M. Polymer nanotechnology: Nanocomposites. Polymer 2008, 49, 3187-3204. [CrossRef]

10. Chivrav, F.; Pollet, E.; Averous, K. Progress in nano-biocomposites based on polysaccharides and nanoclays. Mater. Sci. Eng. R 2009, 67, 1-17. [CrossRef]

11. Bertolino, V.; Cavallaro, G.; Lazzara, G.; Merli, M.; Milioto, S.; Parisi, F.; Sciascia, L. Effect of the biopolymer charge and the nanoclay morphology on nanocomposite materials. Ind. Eng. Chem. Res. 2016, 55, 7373-7380. [CrossRef]

12. Makaremi, M.; Pasbakhsh, P.; Cavallaro, G.; Lazzara, G.; Aw, Y.K.; Lee, S.M.; Milioto, S. Effect of morphology and size of halloysite nanotubes on functional pectin bionanocomposites for food packaging applications. ACS Appl. Mater. Interfaces 2017, 9, 17476-17488. [CrossRef] [PubMed]

13. Zabihi, O.; Ahmadi, M.; Nikafshar, S.; Preyeswary, K.C.; Naebe, M. A technical review on epoxy-clay nanocomposites: Structure, properties, and their applications in fiber reinforced composites. Composites $B$ 2018, 135, 1-24. [CrossRef]

14. Luo, J.J.; Daniel, I.M. Characterization and modeling of mechanical behavior of polymer/clay nanocomposites. Compos. Sci. Technol. 2003, 63, 1607-1616. [CrossRef]

15. Tucker, C.L.; Liang, E. Stiffness predictions for unidirectional short-fiber composites: Review and evaluation. Compos. Sci. Technol. 1999, 59, 655-671. [CrossRef]

16. Tsai, J.; Sun, C.T. Effect of platelet dispersion on the load transfer efficiency in nanoclay composites. J. Compos. Mater. 2004, 38, 567-579. [CrossRef]

17. Weon, J.I.; Sue, H.J. Effects of clay orientation and aspect ratio on mechanical behavior of nylon-6 nanocomposite. Polymer 2005, 46, 6325-6334. [CrossRef]

18. Sheng, N.; Boyce, M.C.; Parks, D.M.; Rutledge, G.C.; Abes, J.I.; Cohen, R.E. Multiscale micromechanical modeling of polymer/clay nanocomposites and the effective clay particle. Polymer 2004, 45, 487-506. [CrossRef] 
19. Zhu, L.J.; Narh, K.A. Numerical simulation of the tensile modulus of nanoclay-filled polymer composites. J. Polym. Sci. 2004, 42, 2391-2406. [CrossRef]

20. Dai, Y.; Mai, Y.W.; Ji, X. Predictions of stiffness and strength of nylon 6/MMT nanocomposites with an improved staggered model. Compos. Part B 2008, 39, 1062-1068. [CrossRef]

21. Dong, Y.; Bhattacharyya, D. A simple micromechanical approach to predict mechanical behavior of polypropylene/organoclay nanocomposites based on representative volume element (RVE). Comput. Mater. Sci. 2010, 49, 1-8. [CrossRef]

22. Hu, H.; Onyebueke, L.; Abatan, A. Characterizing and modeling mechanical properties of nanocomposites-review and evaluation. J. Miner. Mater. Charact. Eng. 2010, 9, 275-319. [CrossRef]

23. Logan, J.D. Applied Mathematics, 2nd ed.; Wiley: New York, NY, USA, 1997.

(C) 2017 by the authors. Licensee MDPI, Basel, Switzerland. This article is an open access article distributed under the terms and conditions of the Creative Commons Attribution (CC BY) license (http:/ / creativecommons.org/licenses/by/4.0/). 